\title{
Shallow Impurity Absorption Spectroscopy in Isotopically Enriched Silicon
}

-M. Steger, A. Yang, D. Karaiskaj and M. L. W. Thewalt

Department of Physics, Simon Fraser University, Burnaby, British Columbia, Canada V5A $1 S 6$

E. E. Haller' , J. W. Ager III' ${ }^{1}$ M. Cardona ${ }^{2}$, H. Riemann ${ }^{3}$, N. V. Abrosimov ${ }^{3}$, A. V. Gusev ${ }^{4}$

A. D. Bulanov ${ }^{4}$, A. K. Kaliteevskii ${ }^{5}$, O. N. Godisov ${ }^{5}$, P. Becker ${ }^{6}$, H.-J. Pohl ${ }^{1}$, K. M. Itoh

${ }^{1}$ UC Berkeley and LBNL, Berkeley, California, USA ${ }^{2}$ Max-Planck-Institut für Festkörperforschung, Stuttgart, Germany: ${ }^{3}$ Institut für Kristallzucht, Berlin, Germany

${ }^{4}$ Institute of Chemistry of High-Pure Substances of the RAS, Nizhny Novgorod, Russian Federation: ${ }^{5}$ Science and Technical Center "Centrotech-ECP", Electrochemical Plant, Saint Petersburg,

Russian Federation; ${ }^{6}$ Physikalisch Technische Bundesanstalt, Braunschweig, Germany: ${ }^{7}$ VITCON Projectconsult GmbH, Jena, Germany: ${ }^{8}$ Department of Applied Physics, Keio University, Yokohama, Japan

\section{Introduction}

Karaiskaj et al. [1] showed that the isotopic randomness present in natural $\mathrm{Si}\left({ }^{\text {nat }} \mathrm{Si}\right)$ causes a significant inhomogeneous broadening of many of the long-studied ground state to excited state infrared absorption transitions of the shallow donor phosphorous and acceptor boron. This was surprising since it was thought that the observed linewidths of shallow impurities in silicon are at their fundamental lifetime limit.

We report improved high-resolution infrared absorption studies of these impurities in isotopically enriched ${ }^{28} \mathrm{Si},{ }^{29} \mathrm{Si}$ and ${ }^{30} \mathrm{Si}$. The new data improves on the linewidths of earlier spectra $[1,2]$ particularly in higher excited states due to reduced concentration broadening. Some of the transitions in ${ }^{28} \mathrm{Si}$ show the narrowest full-width at halfmaximum (FWHM) ever reported for shallow donor and acceptor absorption transitions.

\section{Experimental Method}

As compared to previous studies [1,2] we use improved samples:

- ${ }^{28}$ Si: higher isotopic enrichment, lighter doping

- ${ }^{29} \mathrm{Si} \&{ }^{30} \mathrm{Si}$ : higher chemical purity, (but lower isotopic enrichment) While the chemical purity of the enriched samples is improved, it does not match the purity of ultra-high-purity (UHP) natural silicon samples.

\begin{tabular}{r|rrr|cc} 
& ${ }^{28} \mathrm{Si}$ & \multicolumn{1}{c}{${ }^{29} \mathrm{Si}$} & \multicolumn{1}{c}{${ }^{30} \mathrm{Si}$} & {$[\mathrm{P}]$} & {$[\mathrm{B}]$} \\
${ }^{28} \mathrm{Si}$ & $99.991 \%$ & $0.0075 \%$ & $0.0015 \%$ & $2 \times 10^{12}$ & $5 \times 10^{13}$ \\
${ }^{29} \mathrm{Si}$ & $4.32 \%$ & $91.37 \%$ & $4.30 \%$ & $2 \times 10^{13}$ & $5 \times 10^{13}$ \\
${ }^{30} \mathrm{Si}$ & $2.50 \%$ & $7.70 \%$ & $89.80 \%$ & $2 \times 10^{13}$ & $9 \times 10^{13}$ \\
${ }^{\text {nat }} \mathrm{Si} \mathrm{B}$ & $92.2 \%$ & $4.7 \%$ & $3.1 \%$ & - & $3 \times 10^{14}$ \\
${ }^{\text {nat }} \mathrm{Si}$ S (UHP) & $92.2 \%$ & $4.7 \%$ & $3.1 \%$ & $3 \times 10^{12}$ & $3 \times 10^{12}$ \\
\hline
\end{tabular}

Samples: Freely suspended in sample chamber filled with

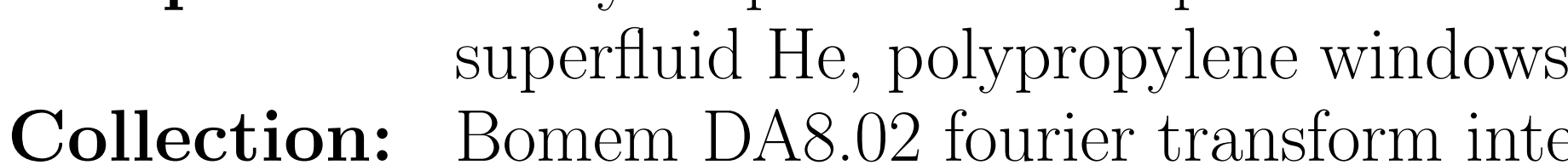

Detector: Silem DA8.02 fourier transform interferometer.

Detector: Silicon composite bolometer at $1.6 \mathrm{~K}$ and

Resolution: Instrumental resolution $0.012 \mathrm{~cm}^{-1}$

\section{Natural Silicon}

Phosphorous

For the donor
phosphorous in

phosphorous in

natural Si we re-

and higher excited

states than shown
before $[3,4,5,6]$.

The $2 p_{0}$ absorp-

tion line has a full

width at half max-

imum (FWHM) of

$0.082 \mathrm{~cm}^{-1}$ and the
$7 p_{+}$has a FWHM

of only $0.057 \mathrm{~cm}^{-1}$

Our sample also

shows two absorp-
tion lines for which

we could not whign fint states anl are the fore labeled ta' and 'b". All other labels were assigned according to Pajot et al. [7].

\section{Boron}

We were also able

to obtain an im-

proved spectrum of

the acceptor boron

showing higher ex-

cited states than

published before [8].

Due to the high
number of different

number of different
labeling schemes we

attempted to assign

labels of theoreti-

cally calculated ex-

cited states as given

by Lewis et al.

Our spectrum clearly shows boron absorption lines up to the $9 \Gamma_{7}$ (labeled 11 in [9]) and $10 \Gamma_{6}$ transitions. Higher energy lines, which could not be assigned to a theoretical level were labeled 'B'.
Isotopically Enriched ${ }^{28} \mathrm{Si}$

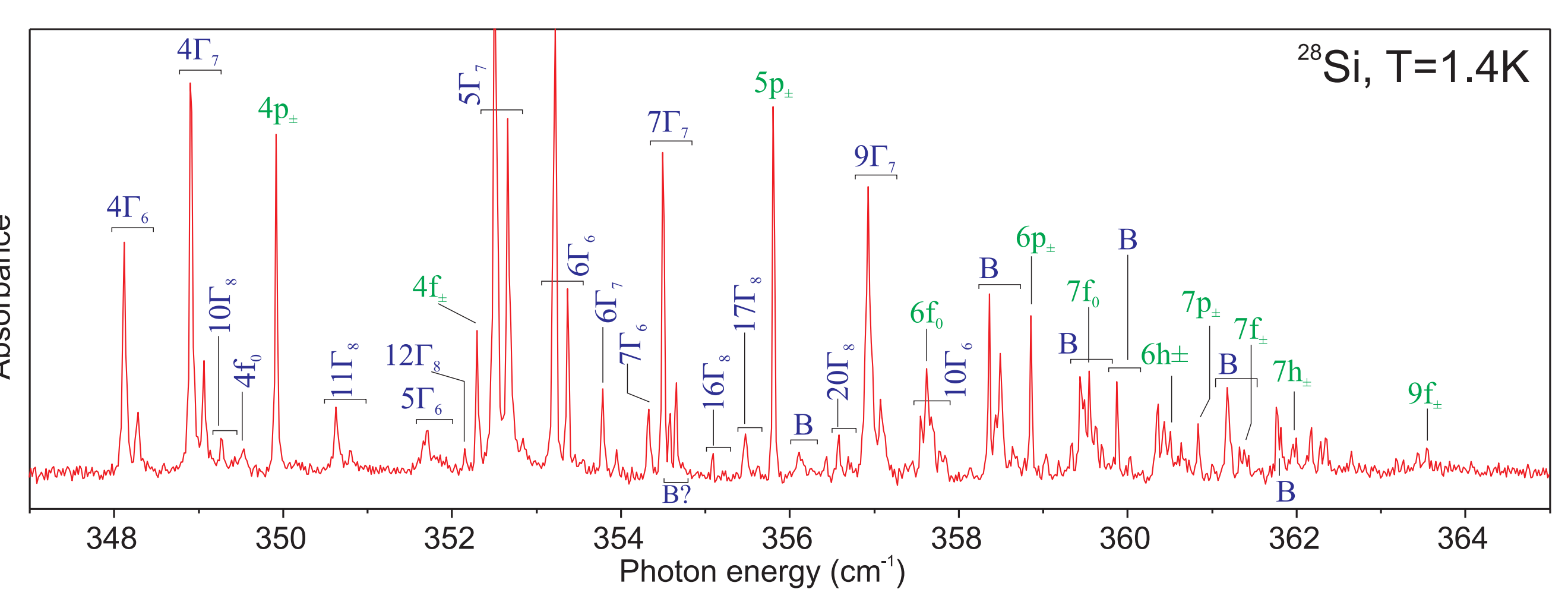

\section{Phosphorous}

In previous publications [1] on isotopically enriched ${ }^{28} \mathrm{Si}$ mainly the low energy absorption lines of the donor phosphorous were found to be sharper than in ${ }^{\text {nat }} \mathrm{Si}$ due to concentration broadening of higher excited states. Here we report narrower linewidths for many of the high energy transitions.

\section{Boron}

Thanks to the reduction of concentration broadening we have observed narrower absorption lines for the acceptor boron, as well.

Many of the boron transitions reveal a $0.15 \mathrm{~cm}^{-1}$ splitting in ${ }^{28} \mathrm{Si}$ which was attributed to the difference in binding energy between ${ }^{10} \mathrm{~B}$ and ${ }^{11} \mathrm{~B}$ acceptors [1]. The doublet intensity ratio reflects the ${ }^{11} \mathrm{~B} /{ }^{10} \mathrm{~B}$ natural abundance ratio of $\sim 80 / 20$

\section{\begin{tabular}{llll|lll}
$2 p_{0}$ & $4 p_{ \pm}$ & $\mathbf{5} \boldsymbol{p}_{ \pm}$ & $6 p_{ \pm}$ & $3 \Gamma_{7}$ & $4 \Gamma_{6}$ & $\mathbf{6} \boldsymbol{\Gamma}_{\mathbf{6}}$
\end{tabular}}

\begin{tabular}{ccccc|cccc} 
& $2 p_{0}$ & $4 p_{ \pm}$ & $\mathbf{5} \boldsymbol{p}_{ \pm}$ & $6 p_{ \pm}$ & $3 \Gamma_{7}$ & $4 \Gamma_{6}$ & $\mathbf{6 \Gamma}_{\mathbf{6}}$ & $7 \Gamma_{7}$ \\
\hline observed & 0.033 & 0.029 & $\mathbf{0 . 0 2 2}$ & 0.023 & 0.048 & 0.040 & $\mathbf{0 . 0 2 5}$ & 0.025
\end{tabular}

\begin{tabular}{l|lllllllll} 
actual & 0.031 & 0.026 & $\mathbf{0 . 0 1 9}$ & 0.020 & 0.046 & 0.038 & $\mathbf{0 . 0 2 2}$ & 0.022 \\
\hline
\end{tabular}

\begin{tabular}{l|l|l} 
vs. ${ }^{\text {nat }} \mathrm{Si}$ & up to $5 \times$ narrower $^{a}$ & up to $10 \times$ narrower $^{b}$ \\
\hline
\end{tabular}

FWHM for selected lines in ${ }^{28} \mathrm{Si}$ in $\mathrm{cm}^{-1}$. The actual FWHM takes the instrumental resolution of

and b) $[8]$.

\section{Line Broadening}

The broadening seen in ${ }^{\text {nat }} \mathrm{Si}$ or any sample of mixed isotopic composition is dominated by an effect which is independent of the small shifts in binding energy between pure ${ }^{28} \mathrm{Si},{ }^{29} \mathrm{Si}$ and ${ }^{30} \mathrm{Si}$ (see below).

The wave function of the ground state is relatively compact, so in samples with mixed isotopes individual impurities can have signigicantly differen local isotopic compositions. These fluctuations induce shifts (and splitting for acceptors) of the ground states, which can be related to the known shift of valence and conduction band energies with average isotopic composition. The excited state wave functions are much more extended and therefore

The difference in isotopic composition sampled by excited and ground state results in inhomogeneous broadening. The valence band shifts acceptor $\mathrm{B}$ the conduction band 10

\section{Comparing ${ }^{28} \mathrm{Si}$ and ${ }^{\text {nat }} \mathrm{Si}$}

For comparison the high energy end of the absorption spectra of ${ }^{28} \mathrm{Si}$ and

${ }^{\text {nat }} \mathrm{Si}$ are overlayed here

The line sharpening and

the additional detail as

well as the shift of the

can be seen

We suspect that the line

labeled $9 \Gamma_{7}$ is actually

${ }^{11} \mathrm{~B} /{ }^{10} \mathrm{~B}$ doublets.

The second spectrum shows that some absorption lines reveal the ${ }^{11} \mathrm{~B} /{ }^{10} \mathrm{~B}$ splitting while

others are dominated

by lifetime broadening

even in ${ }^{\text {nat }} \mathrm{Si}$. Some

transitions like $1 \Gamma_{8}$ show

a significant lifetime

broadening with no
further reduction of their

linewidths in ${ }^{28} \mathrm{Si}$.

This lifetime broaden-

ing effect was explained

by Kane [11] and con-

firmed by Barrie and

Nishikawa $[12,13]$ as

to other nearby excited

phonons.

\section{Binding Energy Shifts}

Theory

$E_{B}$, the ionization energy for shallow (hydrogenic)

$$
E_{B}=R y \cdot m^{*} / \epsilon_{0}^{2}
$$

While this equation is too simple to provide accurate values of impurity binding energies, it can be used to estimate the shift in $E_{B}$ with isotopic composition from the dependence on $m^{*}$ and $\epsilon_{0}$. Donor and acceptor binding energies scale identically with the dependence of $\epsilon_{0}$ $\mathrm{Si}$, the contribution of both $\epsilon_{0}$ and $m^{*}$ act to increase the

\section{Experiment}

Here we compare the shifts in the binding energy $E_{B}$ among the different samples. Our new, improved data allows us to determine those shifts more accurately than before [2] and shows that the previously determined shifts (old $\delta E$ ) were overestimated due to broadening and splitting of the lines in earlier ${ }^{29} \mathrm{Si}$ and ${ }^{30} \mathrm{Si}$ samples.

\begin{tabular}{llllllll}
$2 p_{ \pm}$ & $6 p_{ \pm}$ & $8 f_{ \pm}$ & $E_{B}$ & $1 \Gamma_{8}$ & $1 \Gamma_{6}$ & $5 \Gamma_{7}$ & $E_{B}$ \\
\hline
\end{tabular} \begin{tabular}{lllllllll}
$\delta E$ & -0.100 & -0.129 & -0.134 & -0.15 & -0.264 & -0.350 & -0.407 & -0.44 \\
\hline
\end{tabular} \begin{tabular}{lrr} 
old $\delta E$ & -0.32 & -0.73 \\
\hline Energy shifts (in $\mathrm{cm}^{-1}$ ) for different transitions between ${ }^{28} \mathrm{Si}$ and ${ }^{30} \mathrm{Si}$ with & ${ }^{28} \mathrm{Si}$
\end{tabular} $E=E\left({ }^{28} \mathrm{Si}\right)$

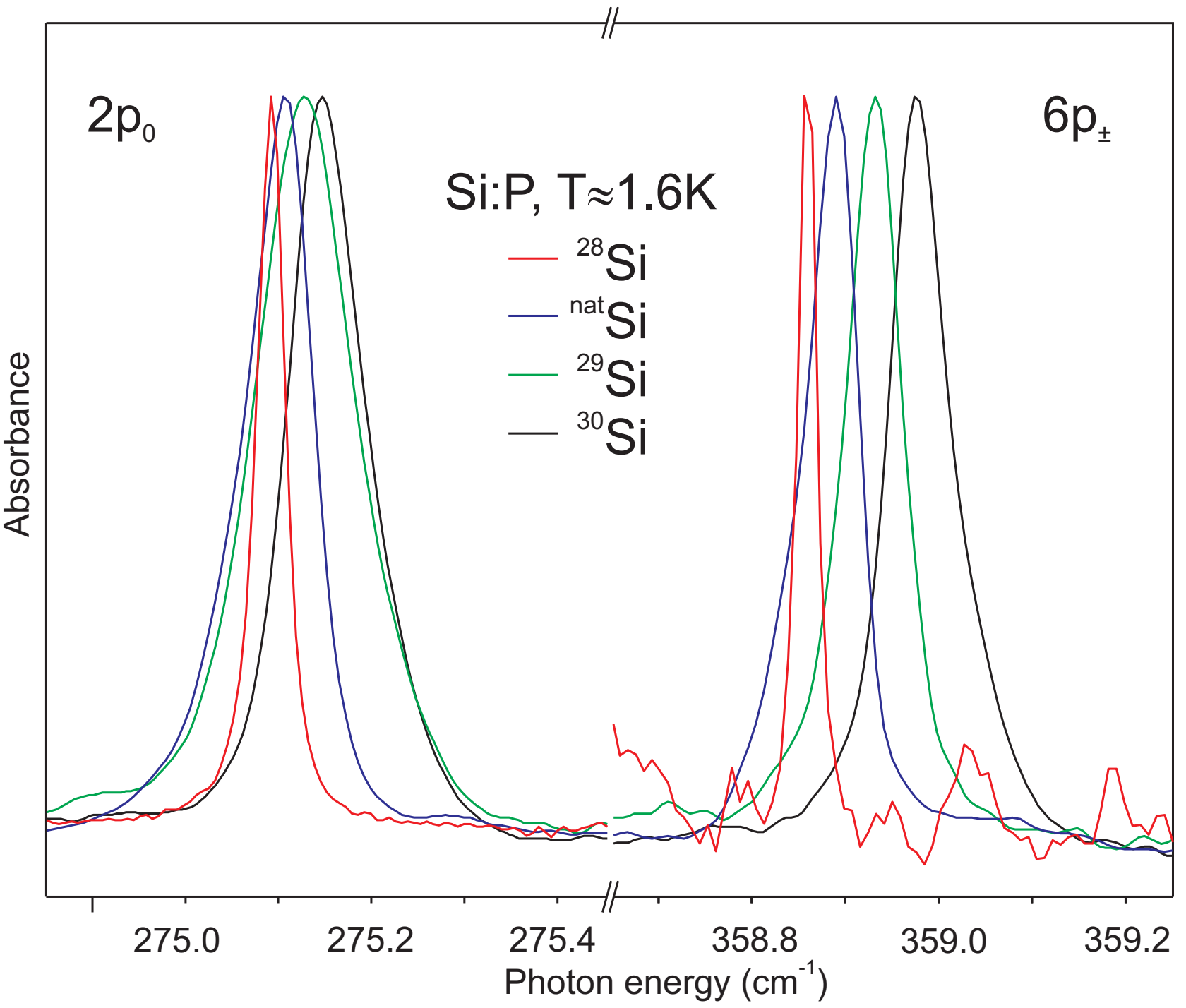

The figures show the shifts for two representative $\mathrm{P}$ and $\mathrm{B}$ transitions in ${ }^{28} \mathrm{Si}^{\text {nat }} \mathrm{Si}$, ${ }^{29} \mathrm{Si}$ and ${ }^{30} \mathrm{Si}$, respectively. The dependence of $\epsilon_{0}$ and $m^{*}$ on the isotopic mass scales the ground state and excited state binding energies by an identical factor, and thus the largest shifts are observed for transitions to the highest excited states.

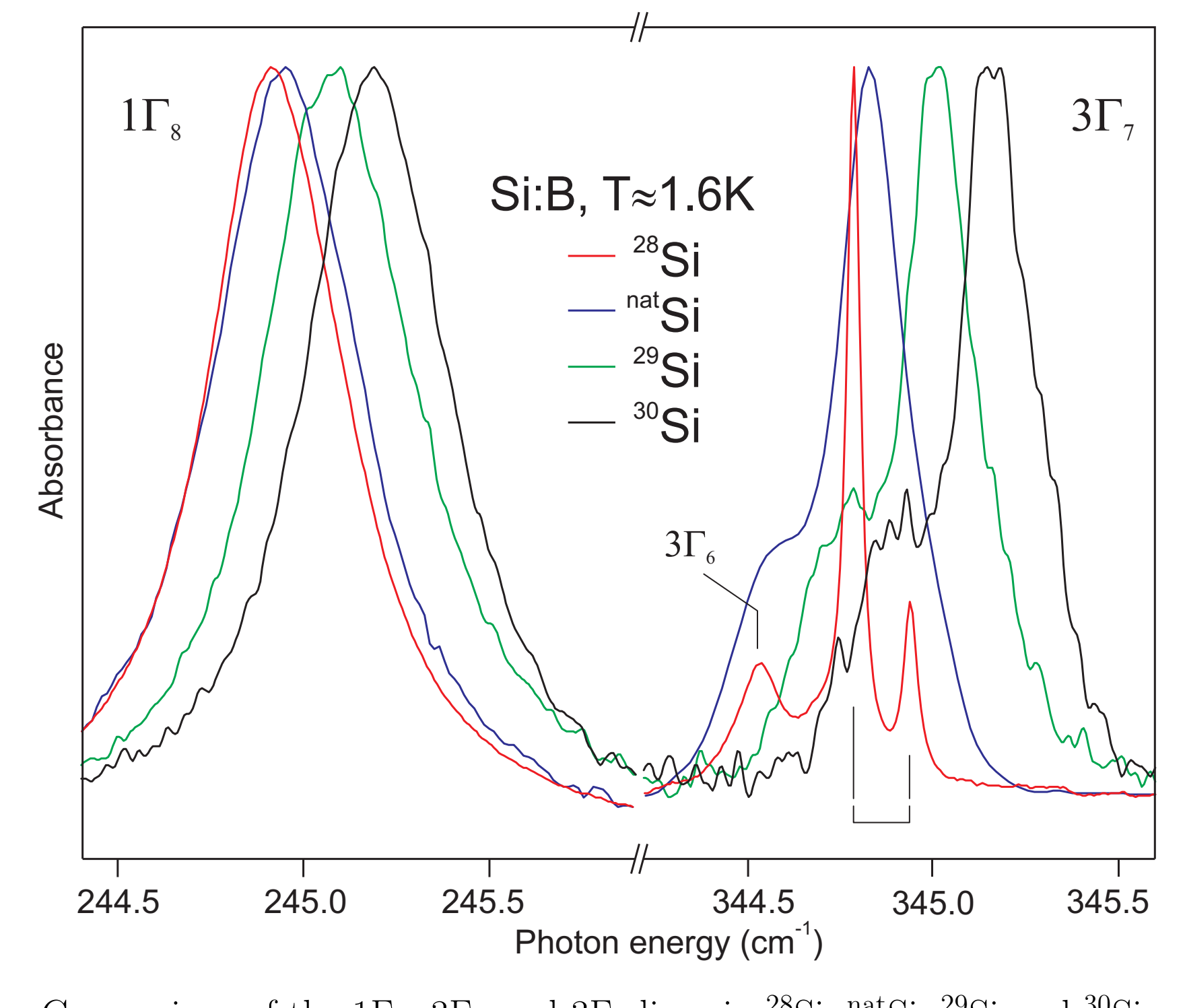

\section{Acknowledgments}

We acknowledge Natural Sciences and Engineering Research Council of Canada (NSERC) for financial support.

\section{References}

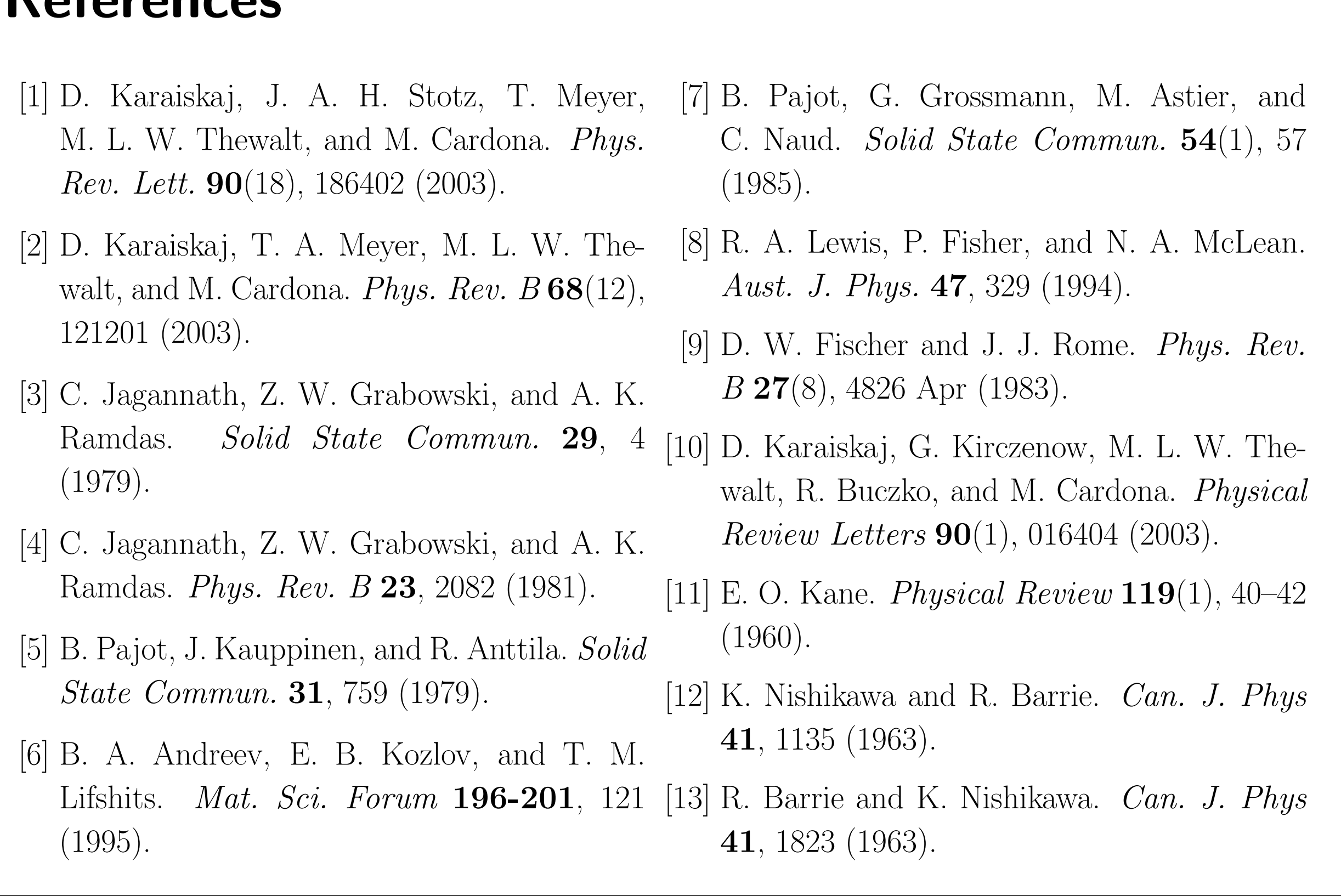

